\title{
Aspectos clínicos, diagnóstico e tratamento do adenocarcinoma pulmonar canino: relato de caso
}

[Clinical aspects, diagnosis, and treatment of canine pulmonary adenocarcinoma: case report]

"Relato de Caso/Case Report"

\section{Lourival Barros de Sousa Brito Pereira ${ }^{1 *}$, Lucilo Bioni da Fonsêca Filho²,} Homero Firmo Pessoa ${ }^{3}$, Priscilla Vírginio de Albuquerque ${ }^{4}$, Nicolli de Albuquerque Leal Gomes D’Alcantara ${ }^{1}$, Júlio Cézar dos Santos Nascimento ${ }^{5}$, Ana Luiza Neves Guimarães Bessa ${ }^{1}$

\author{
${ }^{1}$ Departamento de Medicina Veterinária, Centro Universitário Maurício de Nassau, Recife-PE, Brasil. \\ ${ }^{2}$ Departamento de Medicina Veterinária, Universidade Federal Rural de Pernambuco, Recife-PE, Brasil. \\ ${ }^{3}$ Médico veterinário autônomo, Brasil. \\ ${ }^{4}$ Departamento de Morfologia e Fisiologia Animal, Universidade Federal Rural de Pernambuco, Recife-PE, Brasil. \\ ${ }^{5}$ Departamento de Zootecnia, Universidade Federal Rural de Pernambuco, Recife-PE, Brasil. \\ *Autor para correspondência/Corresponding author: E-mail: lorinho2013.1@ hotmail.com
}

\begin{abstract}
Resumo
A ocorrência de neoplasias pulmonares primárias em cães é rara, acomete principalmente cães idosos, o principal sinal clínico é tosse improdutiva de evolução crônica. Para o diagnóstico, leva-se em consideração achados na anamnese, achados clínicos e exames complementares, sendo a radiografia torácica o principal exame complementar. Para o diagnóstico definitivo é indispensável a realização do exame histopatológico, onde, o adenocarcinoma pulmonar tem sido o tipo mais comumente diagnosticado. $\mathrm{O}$ tratamento mais eficaz é a ressecção cirúrgica do nódulo, podendo associar a quimioterapia como terapia adjuvante. O prognóstico depende do tipo neoplásico, tamanho do tumor, possibilidade em realizar a cirurgia e ausência de metástase. Objetivou-se com este trabalho relatar o caso de um canino, fêmea, de raça Poodle, com onze anos de idade, atendida em uma clínica veterinária, com queixa de tosse há um mês e intolerância ao exercício. No exame físico o animal apresentava-se alerta com discreta taquipneia, taquicardia, mucosas normocoradas. Foram solicitados exames de rotina: hemograma, bioquímica renal e hepática e radiografia torácica, onde, foi identificado uma massa em lobo caudal direito. Para melhor visualização pulmonar, visando um possível tratamento cirúrgico, foi solicitada tomografia computadorizada a qual localizou uma estrutura em lobo medial direito, divergindo da radiografia torácica. $\mathrm{O}$ animal foi encaminhado para toracotomia com acesso intercostal, onde foi realizada lobectomia total do lobo médio direito e o material foi para análise histopatológica fechando o diagnóstico de adenocarcinoma pulmonar. O animal continuou sendo acompanhado por mais oito meses, após a última sessão de quimioterapia não apresentava sinais da doença.
\end{abstract}

Palavras-chave: cirurgia; neoplasia; oncologia; quimioterapia.

\begin{abstract}
The occurrence of primary pulmonary neoplasms in dogs is rare, affects mainly elderly dogs, and the main clinical sign is chronic unproductive cough. For the diagnosis, we take into account findings in the anamnesis, clinical findings, and complementary tests, and chest radiography is the main complementary exam. For the definitive diagnosis, it is indispensable to perform histopathological examination, where pulmonary adenocarcinoma has been the most commonly diagnosed type. The most effective treatment is surgical resection of the nodule, which may be associated with chemotherapy as adjuvant therapy. The prognosis depends on the type of neoplasia, size of the tumor, possibility to perform surgery, and absence of metastasis. The objective of this work was to report the case of a canine, female, Poodle breed, eleven years of age, seen at a veterinary clinic, with complaint of cough a month ago, and intolerance to exercise. On physical
\end{abstract}


examination, the animal was alert with mild tachypnoea, tachycardia, and normal mucous membranes. Routine exams were requested: hemogram, renal biochemistry, hepatic biochemistry, and chest radiography, where a mass in the right caudal lobe was identified. For better pulmonary visualization, aiming at a possible surgical treatment, computed tomography was performed, which located a structure in the right medial lobe, diverging from the chest X-ray. The animal was referred to thoracotomy with intercostal access, where total lobectomy of the right middle lobe was made and the material was submitted to histopathological analysis, establishing the diagnosis of pulmonary adenocarcinoma. The animal continued to be followed-up for another eight months, and after the last chemotherapy session showed no signs of the disease.

Keywords: surgery; neoplasm; oncology; chemotherapy.

\section{Introdução}

Uma neoplasia pode ser interpretada como um "novo crescimento" e pode ser benigna ou maligna. As neoplasias malignas são frequentemente diagnosticadas nas clínicas de pequenos animais, sendo uma causa comum de óbito em cães e gatos (Martins et al., 2011; Rocha et al., 2013; Daleck e De Nardi, 2016).

As afecções pulmonares representam cerca de $4 \%$ dos casos clínicos, em pequenos animais. Pode ser de origem infecciosa, neoplásica primária ou metastática, além daquelas decorrentes de distúrbios circulatórios e afecções pleurais. As neoplasias pulmonares primárias são pouco comuns em cães, no entanto, as metastáticas ocorrem com maior frequência (Ferian et al., 2006; Pereira et al., 2012). São divididas em neoplasias de origem epitelial ou mesenquimal e classificados de acordo com o local de origem (Lucena et al., 2011; Rocha et al., 2013).

A etiologia das neoplasias pulmonares primárias (NPP) é considerada multifatorial, a idade média dos cães portadores é entre 10 e 11 anos e não há predisposição racial, porém, cães da raça Boxer e Dobermann são mais acometidos (Silva et al., 2012). O adenocarcinoma pulmonar é a neoplasia maligna mais diagnosticada representando 70 a $80 \%$ das NPP (Pedroso et al., 2010).

Dentre os exames complementares, o exame radiográfico de boa qualidade, em três projeções, tem sido o mais utilizado na rotina clínica de pequenos animais. Nelas, é possível observar neoplasias com padrões de imagem variável, porém, densidade nodular solitária em lobos caudais é a alteração mais visualizada. A tomografia computadorizada possibilita um estudo mais fidedigno de tamanho, calcificação, número e densidade da lesão. No entanto, para diagnóstico definitivo é necessário a realização do exame histopatológico (Soave et al., 2008; Daleck e De Nardi, 2016).

O tratamento de escolha é a cirurgia, através da exérese da neoplasia. Esta pode ser feita através da lobectomia total ou parcial e pneumectomia do pulmão afetado. $\mathrm{O}$ acesso à cavidade torácica é feito normalmente por toracotomia intercostal ou esternotomia. $\mathrm{O}$ uso de quimioterapia tem sido citado como terapia adjuvante à cirurgia. Os fármacos que podem ser incluídas no protocolo quimioterápico são, cisplatina, carboplatina, etoposídeo, ciclofosfamida, doxorrubicina, sulfato de vincristina, lomustina, ifosfamida, paclitaxel, docetaxel, gencitabina e vinorelbina (Fossum, 2014; Daleck e De Nardi, 2016).

Sendo assim, justifica-se a realização desse trabalho para apresentar um caso de adenocarcinoma pulmonar primário em uma cadela, de raça Poodle, com onze anos de idade, enfatizando as vantagens do diagnóstico precoce e do tratamento cirúrgico associado à quimioterapia adjuvante.

\section{Descrição do Caso}

Foi atendido um canino, fêmea, raça poodle, onze anos de idade com queixa de tosse improdutiva e intolerância ao exercício há um mês. As vacinas anuais estavam em dia, ela não tinha acesso à rua e sua dieta era a base de ração Premium. $\mathrm{O}$ ambiente que o animal vivia não tinha fumante. Ao exame físico, as mucosas estavam normocoradas, escore corporal bom, temperatura retal de $38,9 \mathrm{C}^{\circ}$, não foi notado alterações de ausculta pulmonar e cardíaca apresentando aproximadamente 120 batimentos por minuto (Bpm) e 40 movimentos respiratórios por minuto, no exame da cavidade oral constataram-se placas dentárias gerando uma halitose. Foram solicitados exames de rotina como hemograma, bioquímico e radiografia torácica, a partir disto suspeitou-se de neoplasia pulmonar primária e visto a necessidade de se realizar cirurgia, foi solicitada a tomografia computadorizada para um melhor planejamento 
cirúrgico. Neste exame foi visualizado massa com aspecto neoplásico em hemitórax direito, ao nível do terceiro, quarto e quinto espaço intercostal com dimensões aproximadas de $3,5 \quad \mathrm{~cm} \quad \mathrm{em}$ comprimento x $4,1 \mathrm{~cm}$ em largura x $3,1 \mathrm{~cm}$ em altura e linfonodos intratorácicos com dimensões normais.

No dia da cirurgia para a retirada do lobo médio caudal direito afetado pela neoplasia, foi feito a medicação pré-anestésica com associação de Acepromazina 0,02mg/Kg (Acepran ${ }^{\circledR}, 0,2 \%$ ) e Morfina $0,3 \mathrm{mg} / \mathrm{kg}$ (Dimorf ${ }^{\circledR} 0,1 \mathrm{mg} / \mathrm{mL}$ ) por via intramuscular, após 15 minutos, o animal foi colocado em venóclise e realizou-se uma tricotomia ampla do lado direito do tórax. Na sala cirúrgica recebeu uma pré-oxigenação com $100 \%$ de oxigênio $3 \mathrm{~min}$ antes da indução. Para indução anestésica utilizou-se Midazolan (Dormonid $^{\circledR}$ $5 \mathrm{mg} / 5 \mathrm{ml}$ ), na dose de $0,3 \mathrm{mg} / \mathrm{Kg}$, Quetamina ${ }^{\circledR}$ (Cetamina, $10 \%$ ), na dose de $1 \mathrm{mg} / \mathrm{Kg}$ e Propofol ${ }^{\circledR}$ (Propofol $10 \mathrm{mg} / \mathrm{mL}$ ), na dose de $2 \mathrm{mg} / \mathrm{Kg}$ por via intravenosa, para manutenção utilizou-se Fentanil ${ }^{\circledR}$ (Fentanil $50 \mathrm{mcg} / \mathrm{mL}$ ), na dose de $2 \mathrm{mcg} / \mathrm{Kg}$ em bolus a cada 15 min e isoflurano pela intubação orotraqueal.

$\mathrm{O}$ animal foi posicionado em decúbito lateral esquerdo, visto que o lobo afetado era do lado direito, realizou-se bloqueio loco regional no quinto espaço intercostal com Lidocaína $7 \mathrm{mg} / \mathrm{Kg}$. $\mathrm{O}$ acesso foi feito pelo quinto espaço intercostal, o animal foi mantido em tórax aberto com o auxílio do afastador Finochietto, com ventilação manual assistida. Foram usadas compressas úmidas para separar o lobo lesionado dos demais lobos pulmonares e visualizou-se a veia e artéria pulmonar e o brônquio, em seguida, foi feito a ligadura em massa, com fio náilon 0 , destas estruturas e o lobo pulmonar lesionado foi removido. A cavidade foi preenchida com solução fisiológica morna para visualizar qualquer tipo de vazamento de ar. Não sendo visualizado nenhum tipo de vazamento, foi retirado o soro da cavidade e aproximaram-se as costelas para síntese. Foi mantido um dreno torácico com sonda uretral número 12, durante 3 dias para retornar a pressão negativa do tórax e posteriormente administração de fármacos intrapleural com a finalidade analgésica.

Durante o procedimento, a monitoração do animal anestesiado foi feita por meio de monitor multiparamétrico $\left(\operatorname{Sdamed}^{\circledR}\right)$ na qual avaliou-se frequência cardíaca (FC), frequência respiratória
(FR), SPO2, pressão arterial média (PAM) e eletrocardiograma (ECG). Foi solicitado o internamento do animal por três dias. Para o póscirúrgico foi prescrito Cronidor $^{\circledR}$ (Tramadol 40mg), na dose de $5 \mathrm{mg} / \mathrm{Kg}$ TID por 3 dias, Maxicam $^{\circledR}$ (Meloxicam 2,0mg), na dose de $0,1 \mathrm{mg} / \mathrm{Kg}$ SID por 4 dias, Agemoxi $\mathrm{CL}^{\circledR}$ (Amoxicilina triidratada + Clavulonato de potássio $50 \mathrm{mg}$ ) na dose de $10 \mathrm{mg} / \mathrm{Kg}$ e Dorfin ${ }^{\circledR}$ (Lidocaína $2 \%$ ), na dose de $4 \mathrm{mg} / \mathrm{Kg}$ QID intrapleural por meio de tubo torácico por 3 dias, após o animal retornar da anestesia, o mesmo foi internado em uma clínica veterinária $24 \mathrm{~h}$ para melhores cuidados no póscirúrgico.

O lobo pulmonar foi acondicionado em frasco com formol $10 \%$ e encaminhado para o exame histopatológico. A quimioterapia adjuvante foi iniciada 10 dias depois da retirada dos pontos, os fármacos utilizados foram Carboplatina (Bplatin ${ }^{\circledR} 10 \mathrm{mg} / \mathrm{mL}$ ), na dose de $10 \mathrm{mg} / \mathrm{Kg}$ e Gencitabina $\left(\right.$ Gemzar $^{\circledR}$ 200mg), na dose de 2 $\mathrm{mg} / \mathrm{kg}$, doses de efeito não tóxico.

Antes de cada sessão era feito exame hematológico, o protocolo era constituído de quatro ciclos, cada ciclo era composto por três semanas, no primeiro dia da primeira semana de um ciclo era administrada a gencitabina e carboplatina, no oitavo dia da segunda semana era administrado a gencitabina e na terceira semana era a semana de descanso, como consta na Tabela 1.

A administração da gencitabina e carboplatina era feita da seguinte forma: o animal era colocado em venóclise com solução fisiológica a $0,9 \%$, a administração da gencitabina durava um período de vinte minutos como uma infusão intravenosa lenta. $O$ animal permanecia em fluidoterapia por quatro horas, quando então era feita administração da carboplatina em bolus, na semana que era administrado só a gencitabina o tempo de administração durava um período de vinte minutos através de infusão intravenosa lenta.

O tutor foi orientado a evitar contato direto com as fezes e urina por pelo menos $48 \mathrm{~h}$ após tratamento quimioterápico e voltar com o animal a cada três meses para uma reavaliação clínica e radiográfica.

No primeiro laudo radiográfico de reavaliação, foi identificada uma possível linfomegalia com suspeita de infiltrado neoplásico, causando desvio da traqueia e sem sinais radiográficos sugestivos de neoplasia pulmonar metastática. A confirmação de metástase para o linfonodo, através de citologia, não foi possível 
pelo fato da proprietária não querer dar

continuidade ao diagnóstico.

Tabela 1. Protocolo quimioterápico de associação da gencitabina e carboplatina para tratamento adjuvante do adenocarcinoma pulmonar em cães.

\begin{tabular}{llcc}
\hline Semana de administração & Ciclo & Gencitabina 2mg/Kg IV & Carboplatina $10 \mathrm{mg} / \mathrm{Kg} \mathrm{IV}$ \\
\hline 1 & 1 & $\mathrm{X}$ & $\mathrm{X}$ \\
2 & 1 & $\mathrm{X}$ & \\
3 & 1 & Descanso & Descanso \\
4 & 2 & $\mathrm{X}$ & $\mathrm{X}$ \\
5 & 2 & $\mathrm{X}$ & \\
6 & 2 & Descanso & $\mathrm{X}$ \\
7 & 3 & $\mathrm{X}$ & \\
8 & 3 & Descanso & Descanso \\
9 & 3 & $\mathrm{X}$ & $\mathrm{X}$ \\
10 & 4 & $\mathrm{X}$ & \\
11 & 4 & Descanso & Descanso \\
12 & 4 & & \\
\hline
\end{tabular}

\section{Discussão}

A idade do animal do presente relato era 11 anos, enquadrando-se na faixa etária descrita pela literatura como de maior ocorrência para desenvolver neoplasia pulmonar. Alguns autores relatam que não há predisposição sexual, porém, as fêmeas são mais citadas como de maior ocorrência, assim como encontrado (Leandro et al., 2015).

Embora a literatura cite que as raças mais acometidas são Boxer, Dobermann, Pastor Australiano, Setter Irlandês, Boiadeiro de Berna (Silva et al., 2012) Labrador Retriever, Golden Retriever e Cocker Spaniel (Medeiros et al., 2013), outros autores já relataram em raças diferentes demonstrando que não há predisposição racial. $\mathrm{O}$ animal descrito era um canino de raça Poodle, o que não diverge da literatura, inclusive, por já ter sido citado por Lucena et al. (2010).

$\mathrm{Na}$ anamnese foi constatado que o mesmo não morava em ambiente com fumantes, sendo esse, um dos fatores citados na literatura como contribuinte para o aparecimento das NP (Daleck e De Nardi, 2016).

O sinal clínico mais descrito é a tosse de evolução crônica, o mesmo apresentado pelo animal do presente trabalho (Silva et al., 2012; Daleck e De Nardi, 2016). A radiografia torácica que sugeriu ter a existência de massa neoplásica em lobo caudal direito. Levando em consideração que o mesmo está na faixa etária de risco a ter NP e os sinais clínicos, compatíveis com o que a literatura descreve como mais comum, a suspeita inicial foi de NPP.
A tomografia contribuiu para se ter a localização precisa da neoplasia, que neste caso estava em lobo pulmonar médio direito, divergindo com a indicação de massa em lobo caudal direito sugerida no exame radiográfico, o que teve grande importância no planejamento cirúrgico deste caso (Figura 1) (Daleck e De Nardi, 2016).

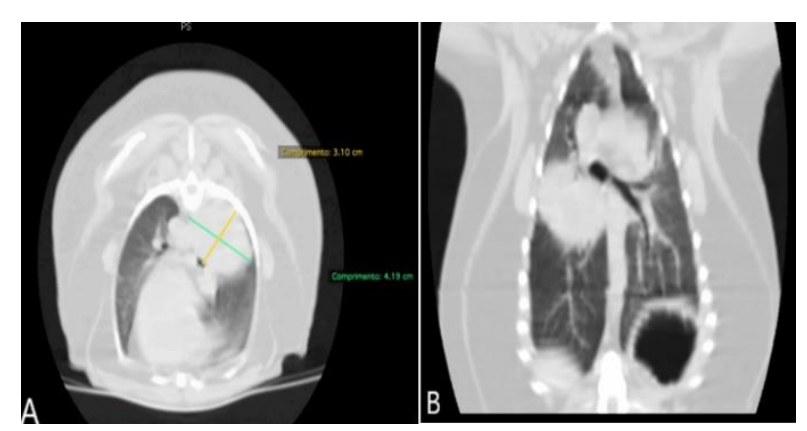

Figura 1. Exame de tomografia computadorizada da cavidade torácica de um cão de 11 anos de idade. A. Corte transversal da cavidade torácica, evidenciando massa de comprimento 3,10 e $4,19 \mathrm{~cm}$. B. Corte longitudinal da cavidade torácica.

O tratamento escolhido foi a cirurgia, visto que a neoplasia era constituída de massa solitária, o animal não apresentava metástase, estava apto para receber anestesia e não apresentava doença concomitante, requisitos estes importantes para se instituir o tratamento cirúrgico (Fossum, 2014; Daleck e De Nardi, 2016). Porém, o tempo de decisão em realizar a cirurgia após o diagnóstico de NP é determinante para eficácia deste tratamento. O que no caso do animal deste trabalho foi prejudicado pela demora em realizar a cirurgia. Foram em torno de trinta e cinco dias de espera até 
a realização da cirurgia, contudo atingiu o objetivo de melhorar a qualidade de vida do animal, deixando-o sem sintomas clínicos, até a última reavaliação.

$\mathrm{O}$ acesso à cavidade torácica, através de toracotomia no quinto espaço intercostal e com a utilização do afastador tipo Finochietto permitiu ótima visibilidade do lobo médio direito. Os lobos adjacentes ao lobo pulmonar médio direito foram isolados com compressas úmidas, com a finalidade de evitar manipulação para não causar embolização pulmonar (Fossum, 2014). Os parâmetros avaliados como $\mathrm{FC}, \mathrm{FR}, \mathrm{SPO}_{2}$, pressão arterial, temperatura retal, PAM e ECG ficaram dentro das normalidades. Os métodos que foram utilizados para minimizar a dor no pós-operatório foram o bloqueio loco regional no espaço intercostal com anestésico local antes de insicionar a pele. No póscirúrgico foi feito lidocaína intrapleural, através do dreno torácico, com Lidocaína 4mg/kg QID, por três dias, realizado no internamento (Tranquilli et al., 2013).

O exame histopatológico feito para se obter um diagnóstico definitivo e se ter mais informações a respeito da NP que o animal apresentava, foi realizado com o material obtido na cirurgia (Figura 2), que mostrou ser uma neoplasia de células epiteliais, mais comumente diagnosticada (Pedroso et al., 2010; Silva, 2012; Copat et al., 2014), com acentuada anisocariose, pleomorfismo celular, pleomorfismo nuclear e 2 a 3 mitoses típicas, características essas que determinam o grau de malignidade da neoplasia. Concluindo ser o adenocarcinoma pulmonar que segundo a literatura é o tipo histológico mais diagnosticado (Pedroso et al., 2010; Silva et al., 2012; Copat et al., 2014).

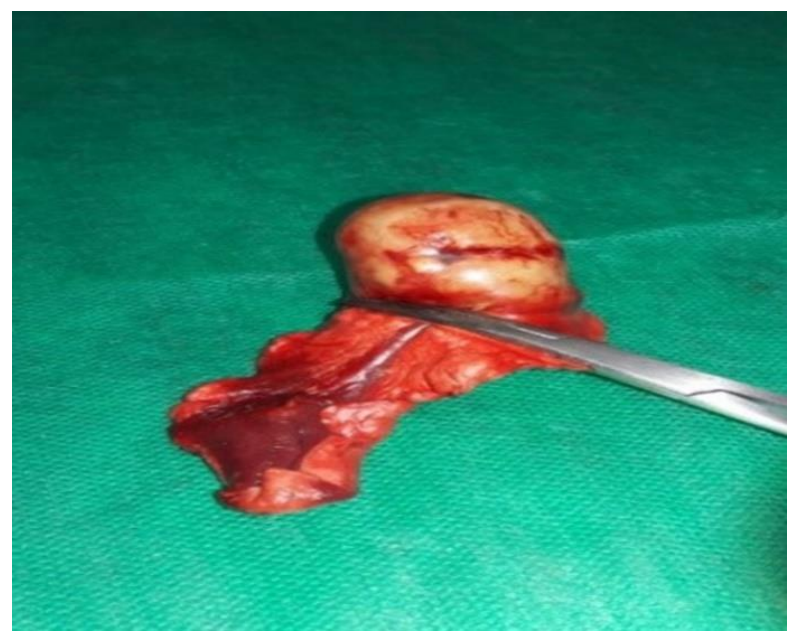

Figura 2. Lobo pulmonar médio direito de um canino de 11 anos de idade, após toracotomia intercostal.
Devido à alta malignidade das NPP, as metástases são frequentes. Sabendo disso, a quimioterapia é uma alternativa de tratamento que visa atuar no controle das recidivas e na progressão das metástases (Daleck e De Nardi, 2016). O animal do presente trabalho foi submetido à quimioterapia adjuvante após a realização da cirurgia, com a finalidade de controle das micrometástases. O uso da Carboplatina, na dose de $10 \mathrm{mg} / \mathrm{kg}$ associado à Gencitabina, na dose de 2 $\mathrm{mg} / \mathrm{kg}$ como terapia quimioterápica adjuvante, mostrou ser um protocolo seguro sem reações adversas. Estes fármacos administrados sozinhos tem o poder de causar mielossupressão. A literatura afirma que a associação das mesmas é bem tolerada nesta dose, porém, toxicidade hematológica, renais e gastrointestinal são comumente relatados o que não foi visto no animal deste trabalho. Os hemogramas feitos antes de cada sessão de quimioterapia, não demonstraram alterações que sugerissem interrupção da mesma, por depressão medular e também não foram observados vômitos nem diarreia, sinais estes que indicariam toxicidade gastrointestinal (Dominguez et al., 2009).

Foi recomendada a tutora que retornasse a cada três meses, pois segundo a literatura, a monitoração periódica após intervenção terapêutica é feita para acompanhar a progressão da neoplasia (Pedroso et al., 2010; Withrow et al., 2012). Essa monitoração é feita por meio de exames de imagem, preferencialmente os mesmos usados no estadiamento inicial, que no caso do animal deste trabalho foi a radiografia torácica, conforme Figuras 3 e 4 .

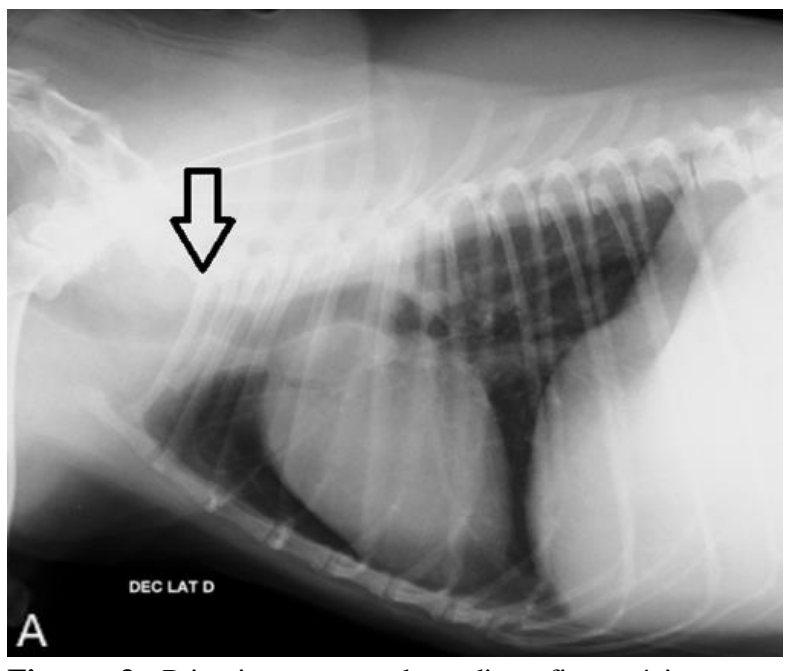

Figura 3. Primeiro exame de radiografia torácica, para monitorização, em canino com 11 anos de idade. Projeção em decúbito lateral direito visualizando um desvio ventrodorsal da traqueia. 


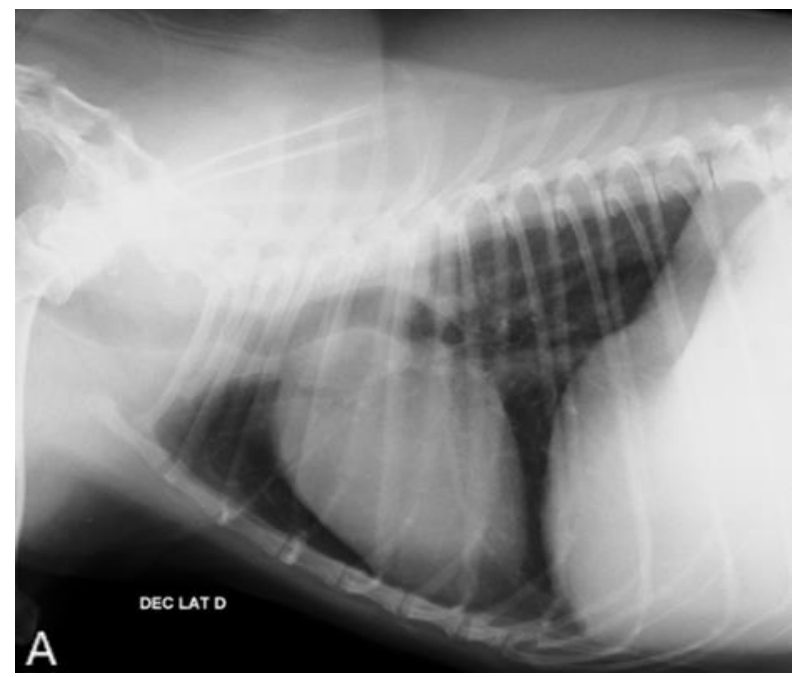

Figura 4. Segundo exame de radiografia torácica, de monitorização, em canino de 11 anos de idade. Projeção em decúbito lateral direito visualizando um desvio ventrodorsal da traqueia.

\section{Conclusão}

Conclui-se, portanto, que as neoplasias pulmonares primárias mesmo consideradas incomuns estão sendo cada vez mais diagnosticadas na clínica de pequenos animais sendo um importante diagnostico diferencial para animais idosos, que chegam à clínica com queixa de tosse crônica. A tomografia computadorizada é um exame bastante sensível para detectar neoplasias mínimas, útil para o diagnóstico precoce e fundamental para um melhor planejamento cirúrgico.

O uso da carboplatina associado à gencitabina como quimioterapia adjuvante mostrou-se ser eficaz no tratamento complementar ao tratamento cirúrgico, bastante seguro nas doses utilizadas, sem causar reações adversas como mielossupressão ou reações gastrointestinais.

\section{Conflito de Interesse}

Os autores declaram não existir conflito de interesse.

\section{Referências}

Copat, B.; Feranti, J.P.S.; Chaves, R.O.; Silva, A.P.; Oliveira, M.T.; Pohl, V.H.; Corrêa, L.F. D.; Brun, M.V. Toracotomia com ressecção de costela para lobectomia pulmonar de adenocarcinoma papilar primário extenso em cão. Acta Scientiae Veterinariae, 42(Suppl 1): 58, 2014.

Daleck, C.R.; De Nardi, A.B. Oncologia em cães e gatos. $2^{a}$ ed. Rio de Janeiro: Roca, 2016. 766p.

Dominguez, P.A.; Dervisis, N.G.; Cadile, C.D.; Sardu, L.; Kitchell, B.E. Combined gemcitabine and carboplatin therapy for carcinomas in dogs. Journal of Veterinary Internal Medicine, 23(1): 130-137, 2009.

Ferian, P.E.; Silva, E.F.; Guedes, R.G.; Tôrres, R.C.S.; Carneiro, R.A. Diagnóstico citológico de neoplasia pulmonar por meio de lavado broncoalveolar em uma cadela: Relato de caso. Arquivo Brasileiro de Medicina Veterinária e Zootecnia, 58(5): 776-780, 2006.

Fossum, T.W. Cirurgia de pequenos animais. $4^{\mathrm{a}}$ ed. Rio de Janeiro: Elsevier, 2014. 1640 p.

Leandro, R.M.; Barbosa, A.; D’Oliveira, K.S.; Quirico, I.A.; Migueis, F.; Vincenzo, T.S. Carcinoma pulmonar adenoescamoso em cão Relato de Caso. Uniciências, 19(2): 155-160, 2015.

Lucena, R.B.; Maia, L.A.; Dantas, A.F.M.; Nobre, V.M.T.; Macedo, J.T.S.A.; Filho, E.M.G.; Barros, C.S.L. Carcinoma bronquíolo-alveolar difuso em um cão: aspectos clínico-patológicos e imunohistoquímicos. Ciência Rural, 41(1): 160-163, 2010.

Martins, D.B.; Teixeira, L.V.; França, R.T.; Lopes, S.T.A. Biologia tumoral no cão: Uma revisão. Medvep - Revista Científica de Medicina Veterinária - Pequenos Animais e Animais de Estimação, 9(31): 630-637, 2011

Medeiros, A.A.; Soares, N.P.; Castro, M.B.; Szabó, M.P.J.; Gudim, L.F.; Oliveira, J.R. Adenocarcinoma acinar pulmonar em um cão Relato de caso. Archives of Veterinary Science, 18(3): 517-519, 2013.

Pedroso, T.C.; Grance, S.R.M.; Babo-Terra, V.J.; Neto, C.S. Adenocarcinoma papilar de pulmão em cão: Relato de caso. Pubvet, 4(34): 938943, 2010.

Pereira, W.L.A.; Monger, S.G.B.; Cardoso, A.M.C. Adenoma papilar pulmonar em cão: relato de caso. Ars Veterinaria, 28(43): 190194, 2012.

Rocha, J.R.; Dias, F.G.G.; Magalhães, G.M.; Santilli, J.; Dias, L.G.G.G. Carcinoma broncoalveolar com metástase intracraniana em cão: Relato de caso. Enciclopédia Biosfera, 9(17): 646-655, 2013.

Silva, E.O.; Green, K.T.; Wasques, D.G.; Reis, A.C.F.; Bracarense, A.P.F.R.L. Tumor primário pulmonar metastático em três cães. Semina: Ciências Agrárias, 33(2): 3271-3278, 2012.

Soave, T.; Sousa, D.P.; Moreno, K.; Beloni, S.N.E.; Gonzales, J.R.M.; Grotti, C.C.B.; Reis, A.C.F. A importância do exame radiográfico torácico na abordagem de animais portadores de 
neoplasias. Semina: Ciências Agrárias, 29(2): 399-406, 2008.

Tranquilli, W.J.; Thurmon, J.C.; Grimm, K.A. Anestesiologia e analgesia veterinária. São Paulo: Roca, 2013. 1216p.
Withrow, S.; Vail, D.; Page, R. Small animal clinical oncology. $5^{\text {th }}$ ed. Philadelphia: Elsevier, 2012. 768p. 